\title{
Teste cutâneo em doenças alérgicas: uma revisão sistemática
}

\author{
Skin test in allergic diseases: a systematic review
}

\author{
Karina Soldera ${ }^{1}$, Bianca Martininghi Bugança ${ }^{1}$, Anelise Santos Oliveira ${ }^{1}$, Caroline Pieta Dias ${ }^{2}$, \\ Paulo Márcio Pitrez ${ }^{3}$, Cristian Roncada ${ }^{4} \bowtie$
}

${ }^{1}$ Graduanda em Biomedicina, Faculdade da Serra Gaúcha (FSG), Caxias do Sul, RS

2 Doutora em Ciências do Movimento Humano pela Universidade Federal do Rio Grande do Sul (UFRGS). Professora da FSG, Caxias do Sul, RS

${ }^{3}$ Doutor em Pneumologia. Diretor do Instituto de Pesquisas Biomédicas da Pontifícia Universidade Católica do Rio Grande do Sul (PUCRS), Porto Alegre, RS

${ }^{4}$ Doutor em Saúde da Criança pela PUCRS. Professor da FSG, Caxias do Sul, RS.

\section{RESUMO}

Objetivos: Identificar os principais alérgenos aplicados em testes cutâneos para sensibilização atópica em pacientes com diagnóstico de asma e rinite alérgica.

Métodos: Uma revisão sistemática foi realizada para identificar estudos clínicos nos últimos 10 anos, tendo como desfecho a utilização do teste cutâneo (prick test) no diagnóstico de asma e rinite alérgica. Foi realizada uma pesquisa nas bases de dados PubMed, Scielo e ScienceDirect. Foram incluídos artigos que abordam a utilização direta do teste cutâneo no diagnóstico das doenças alérgicas. Além disso, os estudos obrigatoriamente deveriam descrever o protocolo de utilização, bem como os alérgenos aplicados no teste. Para seleção dos artigos, três pesquisadores analisaram de forma independente os critérios de inclusão, sendo os mesmos analisados por mais dois revisores.

Resultados: Treze instrumentos foram incluídos na sistemática, tendo uma variação expressiva na quantidade de reagentes (de 3 a 36 tipos). Dos 36, cinco possuíram a maior frequência de positividade à sensibilização (Artemisia vulgaris, Dermatophagoides farinae, Dermatophagoides pteronyssinus, epitélios de cão e de gato). Como parte do protocolo de aplicação, a região do antebraço e o período de 15 a 20 minutos são os mais frequentes.

Conclusões: Alérgenos mais comuns em alergia respiratória foram descritos, sendo importante levar em consideração a região e população investigadas.

DESCRITORES: testes cutâneos; diagnóstico; asma; rinite.

\section{ABSTRACT}

Aims: To identify the major allergens used in skin tests for atopic sensitization in patients with asthma and allergic rhinitis.

Methods: A systematic review was performed to identify clinical studies in the past 10 years whose outcomes included the skin test (prick test) in the diagnosis of asthma and allergic rhinitis. The PubMed, SciELO, and ScienceDirect databases were searched and articles that addressed the direct use of skin testing in the diagnosis of allergic diseases were included. In addition, the studies had to describe the protocol and the allergens used in the test. For selection of the articles, three researchers independently analyzed the inclusion criteria, which were analyzed by two other reviewers.

Results: Thirteen instruments were included in the systematic review, and the amount of reagents used varied significantly (between 3 and 36 ). Of the 36 reagents, five had the highest frequency of positive sensitization (Artemisia vulgaris, Dermatophagoides farinae, Dermatophagoides pteronyssinus, dog and cat epithelia). As part of the application protocol, the forearm and the period of 15-20 minutes are the most frequently used.

Conclusions: The most frequently detected allergens were described, and both the region and the studied population are important factors to be considered.

KEY WORDS: skin tests; diagnosis; asthma; rhinitis. 


\section{INTRODUÇÃO}

A prevalência de doenças respiratórias alérgicas, como é o caso da asma e da rinite, tem aumentado significativamente nas últimas décadas, tornando-se uma grande preocupação médica [1]. As principais causas no aumento dessas doenças derivam-se de fatores ambientais, como poluição do ar e diminuição da exposição a estímulos microbiológicos, além de mudanças nos hábitos alimentares [2].

A alergia, por sua vez, é uma reação imunológica exagerada a proteínas (alérgenos), em especial aos aeroalérgenos (ácaros, plantas, fungos, pelos de animais) [3]. Além desses, outros fatores podem ser responsáveis pelo desenvolvimento de alergia (fatores genéticos, idade média de exposição a alérgenos e número de irmãos) [4].

Para confirmação da presença de alergia, os procedimentos diagnósticos mais comuns são o teste da imunoglobulina $\mathrm{E}$ (IgE) específica e o teste cutâneo (TC) [5]. O TC é o método mais utilizado na área clínica para investigação de sintomas alérgicos, sendo considerado o teste padrão na avaliação de sensibilização a alérgenos [6], sendo um método rápido, simples e bastante acessível. Sua principal desvantagem é a falta de padronização, pois o resultado depende dos tipos de extratos utilizados e da experiência do profissional responsável pela análise. Além disso, sua especificidade varia de acordo com a condição alérgica do paciente [7].

A relação entre os resultados do $\mathrm{TC}$ e da doença alérgica é interpretado pela história clínica do paciente [8,9]. A seleção dos alérgenos deve ser feita com base em sintomas do paciente, exposições ambientais, situação ocupacional e idade [10]. Além disso, a história clínica do paciente deve ser considerada na interpretação do teste de alergia [11]. A determinação dos alérgenos mais comuns e a relação entre os resultados do TC com as doenças alérgicas em cada área desempenha um papel importante no diagnóstico e tratamento de doenças alérgicas, como no caso da imunoterapia $[11,12]$.

A presente revisão sistemática tem como objetivo identificar os principais alérgenos aplicados e identificados em testes cutâneos para sensibilização atópica em pacientes com diagnóstico de asma e rinite alérgica.

\section{MÉTODOS}

Para desenvolvimento da revisão sistemática foi realizada uma busca nas bases de dados PubMed,
ScienceDirect e SciELO, no período de setembro e outubro de 2014, identificando estudos que utilizaram o teste cutâneo (prick test) para sensibilização atópica em pacientes com asma e rinite alérgica. Como critérios de inclusão, os artigos deveriam consistir em estudos originais nos últimos 10 anos, sendo descrito o protocolo utilizado para avaliação do desfecho, incluindo tipo de teste, local de aplicação, tempo de exposição, materiais e alérgenos utilizados. Artigos sem essas informações não foram incluídos, assim como os artigos que não estavam disponíveis na íntegra para leitura.

Como estratégia de busca foi adotada uma pesquisa baseada em palavras-chave específicas na língua inglesa (EN-USA), com auxilio de operadores booleanos (AND e OR), além de parênteses para ordenação dos operadores e aspas para identificação de palavras compostas, da seguinte forma: (( "skin $\equiv$ test" OR "prick test" OR "skin prick test" OR SPT) AND Rhinitis AND Asthma). As palavras-chave foram aplicadas nos campos de busca Título (Title), Resumo (Abstract) e Palavras-chave (Key words), para evitar a inclusão excessiva de artigos. Dessa forma, as palavras-chave deveriam estar em pelo menos um dos três campos de busca. Além dos critérios descritos não foram aplicados filtros adicionais, como, por exemplo, idioma do artigo ou público alvo. Para auxilio na identificação de estudos duplicados, incluídos ou excluídos, exportamos as buscas das bases de dados nos formatos/extensões (*.ris, *.bibtex e *.medline), sendo importadas para o software específico para elaboração de revisões sistemáticas StArt (State of the Art through Systematic Review) [13]. Mediante exportações, tais análises foram realizadas por três pesquisadores (separadamente) e avaliadas por mais dois revisores.

Como critério de relevância nas escolhas, foram adotadas três etapas para inclusão e exclusão dos artigos, sendo estes: a) artigos selecionados igualmente pelos três pesquisadores foram incluídos automaticamente; b) artigos selecionados por apenas um pesquisador foram analisados por um revisor; c) artigos incluídos por dois pesquisadores ou no caso de apenas um, mas com inclusão após análise do revisor, foram discutidos pelo grupo, caso a caso, para finalização do processo.

A qualidade metodológica dos estudos primários foi avaliada pelo sistema GRADE que foi proposto pelo grupo Grades of Recommendation, Assessment, Development and Evaluation [14]. Além disso, após a seleção dos estudos, foi aplicado um check list contendo perguntas (Quadro 1) para verificar o nível de significância e inferência científica dos mesmos. 
Quadro 1. Avaliação metodológica seguindo os critérios GRADE.

\begin{tabular}{|c|c|c|c|c|c|c|c|c|c|c|c|c|c|}
\hline Parâmetros avaliados & 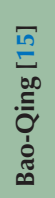 & 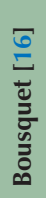 & 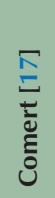 & 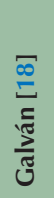 & 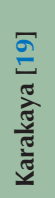 & 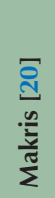 & 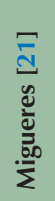 & 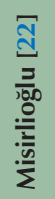 & 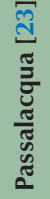 & 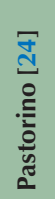 & 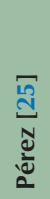 & 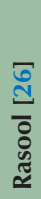 & 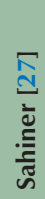 \\
\hline 1. Os desfechos apresentados são relevantes cientificamente? & $\mathrm{S}$ & $\mathrm{S}$ & $\mathrm{S}$ & S & $\mathrm{S}$ & $\mathrm{S}$ & $\mathrm{S}$ & S & $\mathrm{S}$ & S & $\mathrm{S}$ & $\mathrm{S}$ & $\mathrm{S}$ \\
\hline 2. A metodologia é descrita de forma clara e condiz com o desfecho principal? & $\mathrm{S}$ & $\mathrm{S}$ & $\mathrm{S}$ & $\mathrm{S}$ & S & $\mathrm{S}$ & S & S & $\mathrm{S}$ & $\mathrm{S}$ & $\mathrm{S}$ & $\mathrm{S}$ & $\mathrm{S}$ \\
\hline $\begin{array}{l}\text { 3. Como desfecho principal, utilizam o teste cutâneo no diagnóstico clínico } \\
\text { para asma e rinite alérgica? }\end{array}$ & $\mathrm{S}$ & $\mathrm{S}$ & $\mathrm{S}$ & $\mathrm{S}$ & $\mathrm{S}$ & $\mathrm{S}$ & $\mathrm{S}$ & $\mathrm{S}$ & $\mathrm{S}$ & $\mathrm{S}$ & $\mathrm{S}$ & $\mathrm{S}$ & $\mathrm{S}$ \\
\hline 4. Há outra associação com o desfecho principal? & $\mathrm{N}$ & $\mathrm{N}$ & $\mathrm{N}$ & $\mathrm{N}$ & $\mathrm{S}$ & $\mathrm{N}$ & $\mathrm{S}$ & $\mathrm{S}$ & $\mathrm{S}$ & $\mathrm{N}$ & $\mathrm{N}$ & $\mathrm{N}$ & $\mathrm{N}$ \\
\hline 5. Há um grupo controle para comparar o desfecho principal? & $\mathrm{N}$ & $\mathrm{N}$ & $\mathrm{N}$ & $\mathrm{N}$ & $\mathrm{S}$ & $\mathrm{N}$ & $\mathrm{S}$ & $\mathrm{N}$ & $\mathrm{S}$ & $\mathrm{N}$ & $\mathrm{N}$ & $\mathrm{N}$ & $\mathrm{N}$ \\
\hline 6. A amostra é estatisticamente significativa? & $\mathrm{S}$ & $\mathrm{S}$ & $\mathrm{S}$ & $\mathrm{S}$ & $\mathrm{S}$ & $\mathrm{S}$ & $\mathrm{S}$ & $\mathrm{S}$ & $\mathrm{S}$ & $\mathrm{S}$ & $\mathrm{S}$ & $\mathrm{S}$ & $\mathrm{S}$ \\
\hline 7. Os autores expressam de forma clara os resultados? & $\mathrm{S}$ & $\mathrm{S}$ & $\mathrm{S}$ & $\mathrm{S}$ & $\mathrm{S}$ & $\mathrm{S}$ & $\mathrm{S}$ & $\mathrm{S}$ & $\mathrm{S}$ & $\mathrm{S}$ & $\mathrm{S}$ & $\mathrm{S}$ & $\mathrm{S}$ \\
\hline 8. Os resultados vão de encontro com a hipótese? & $\mathrm{S}$ & $\mathrm{S}$ & $\mathrm{S}$ & $\mathrm{N}$ & $\mathrm{S}$ & $\mathrm{S}$ & $\mathrm{S}$ & $\mathrm{S}$ & $\mathrm{S}$ & $\mathrm{S}$ & $\mathrm{S}$ & $\mathrm{S}$ & $\mathrm{S}$ \\
\hline 9. Os autores expressam as limitações do estudo? & $\mathrm{N}$ & $\mathrm{N}$ & $\mathrm{N}$ & $\mathrm{N}$ & $\mathrm{N}$ & $\mathrm{N}$ & $\mathrm{N}$ & $\mathrm{N}$ & $\mathrm{N}$ & $\mathrm{N}$ & $\mathrm{N}$ & $\mathrm{N}$ & $\mathrm{N}$ \\
\hline 10. A conclusão traz alguma inferência científica? & $\mathrm{S}$ & $\mathrm{S}$ & $\mathrm{S}$ & $\mathrm{S}$ & $\mathrm{S}$ & $\mathrm{S}$ & $\mathrm{S}$ & $\mathrm{S}$ & $\mathrm{S}$ & $\mathrm{S}$ & $\mathrm{S}$ & $\mathrm{S}$ & $\mathrm{S}$ \\
\hline 11. Os potenciais conflitos de interesse são declarados? & NC & $\mathrm{S}$ & NC & NC & $\mathrm{NC}$ & NC & NC & NC & NC & NC & NC & $\mathrm{S}$ & $\mathrm{S}$ \\
\hline
\end{tabular}

CO: Coorte; CC: Caso-Controle; TR: Transversal; S: Sim; N: Não; NC: Não consta

\section{RESULTADOS DA SELEÇÃO}

No total, foram encontrados por meio da busca eletrônica 856 artigos (PubMed 489; ScienceDirect 348; SciELO 19). Inicialmente, 74 artigos não foram selecionados em virtude de duplicidade e 660 por não se enquadrarem no tema proposto. Assim, foram préselecionados 122 artigos; destes, 99 foram excluídos por não se enquadrarem nos critérios de inclusão e 10 (seis do PubMed e quatro do ScienceDirect) por não estar acessível o texto completo. Assim, foram incluídos para análise 13 estudos originais (Figura 1).

Para melhor entendimento da revisão sistemática, os estudos são resumidos e demonstrados da seguinte forma: avaliação dos critérios de qualidade metodológica pelo método GRADE (Quadro 1); dados gerais e caracterização dos estudos incluídos (Tabela 1); identificação dos reagentes mais utilizados nos testes cutâneos em casos de asma e rinite alérgica (Tabela 2).

\section{CONTEÚDO DA REVISÃO}

$\mathrm{Na}$ avaliação metodológica (Quadro 1), os 13 artigos demonstraram boa qualidade, com foco central na aplicação do teste cutâneo para auxilio no diagnóstico de doenças alérgicas e/ou quantidade e grau de sensibilidade dos reagentes para identificação dos alérgenos mais sensíveis e precisos para serem aplicados nos testes cutâneos. Apenas o estudo de

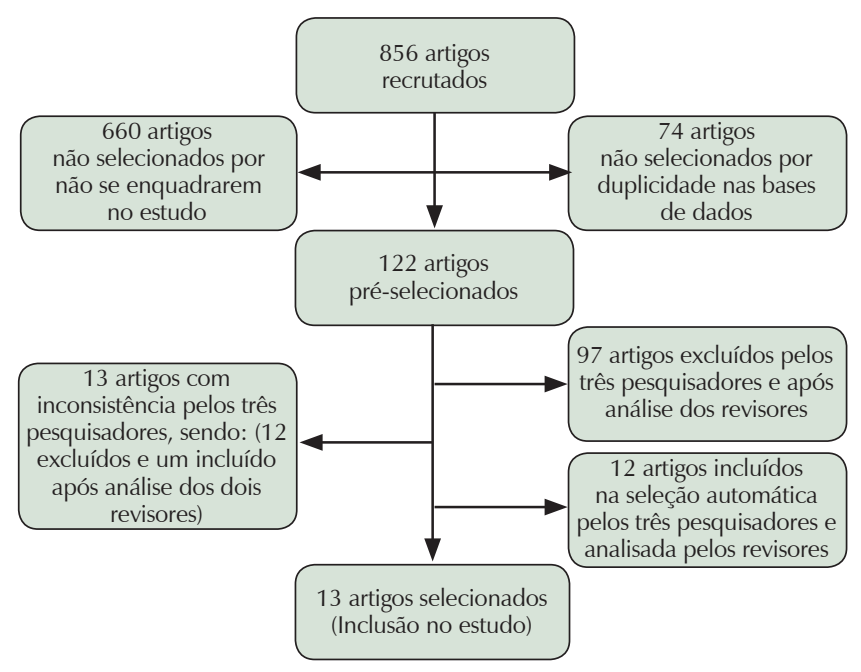

Figura 1. Fluxograma de seleção dos artigos. Busca nas bases de dados PubMed, ScienceDirect e SciELO, no período de setembro e outubro de 2014, identificando estudos que utilizaram o teste cutâneo (Prick test) para sensibilização atópica em pacientes com asma e rinite alérgica.

Galván et al. [18], que avaliou a associação entre determinados alérgenos e o período sazonal, não encontrou relação entre os resultados e a hipótese inicial.

Na Tabela 1 são ilustradas as principais caracterizações dos estudos, demonstrando que o teste cutâneo é preferencialmente aplicado na superfície volar do antebraço, com protocolo de sensibilização de 15 a 20 minutos de duração. Parece não haver um consenso 
sobre a quantidade necessária para aplicação dos reagentes, cuja variedade é alta (de 3 a 36 diferentes tipos de alérgenos). Os principais alérgenos utilizados são os epitélios de cão e gato, acompanhados de duas espécies de Dermatophagoides (D. farinae e D. pteronyssinus) e uma de planta (Artemisia vulgaris), conforme demonstrado na Tabela 2.

Bao-Qing et al. [15] desenvolveram um estudo em 6.304 pacientes chineses, com o objetivo de investigar a prevalência de baratas no continente da China e da reatividade cruzada de $\operatorname{IgE}$ entre alérgenos de barata, poeira e ácaros. A histamina $(10 \mathrm{mg} / \mathrm{ml})$ foi usada como controle positivo e solução salina foi usada como controle negativo. Os autores não especificaram local de aplicação, tempo de duração do teste e tipo de lanceta utilizada. O estudo concluiu que $25,7 \%$ dos pacientes apresentaram reação a barata americana (Periplaneta americana) e 18,7\% a barata alemã (Blattella germanica). O percentual positivo de reação alérgica a barata foi maior no sul do que no norte da China, maior em adultos do que em crianças, e maior no sexo masculino do que no feminino [15].

Bousquet et al. [16] desenvolveram um estudo para definir o número e o tipo de alérgenos necessários para identificar um paciente como sensibilizado. Fizeram o teste em 2.928 pacientes, sendo aplicados os seguintes alérgenos: Alternaria, Aspergillus fumigatus, Cladosporium herbarum, epitélio de gato e cão, Dermatophagoides pteronyssinus, Dermatophagoides farinae, Blatella, pólens de amieiro, Ambrosia, Artemisia, bétula, cipreste, avelã, plátano, oliva, mistura de grama, prado aveia, pé de grama do galo, festuca, azevém perene, grama Timothy, prado liso e Parietaria. Utilizaram histamina como controle positivo e solução salina como controle negativo. Os autores não especificaram o local de aplicação, tempo de duração do teste e tipo de lanceta utilizada. Os resultados foram registrados após 15 minutos. O diâmetro da pápula para ser considerado positivo foi de $\geq 3 \mathrm{~mm}$. Indivíduos com pelo menos um teste cutâneo positivo foram considerados como sensibilizados. Os autores concluíram que a prevalência de sensibilização variou de $37,1 \%$ quando apenas um alérgeno foi testado. Pólen de gramíneas foi o mais prevalente (68,2\%) e oito alérgenos dos 18 (pólen de gramíneas, Dermatophagoides pteronyssinus, vidoeiro pólen, epitélio de gato, artemísia, pólen de oliveira, Blatella e Alternaria) permitiram a identificação de mais de 95\% dos indivíduos sensibilizados [16].

Comert et al. [17] desenvolveram um estudo com objetivo de identificar ao menos $95 \%$ de pessoas sensibilizadas com sintomas de asma e/ou rinite. Aplicaram o teste cutâneo em 7.492 pacientes, sendo aplicados os seguintes alérgenos: Patense phleum, Dermatophagoides pteronyssinus, Olea europaea, Artemisia vulgaris, Corylus avellana, Betula verrucosa, Parietaria officinalis, Populus alba, Aspergillus fumigatus, epitélios de gato, cachorro e cavalo, Alternaria alternata, Cladosporium herbarum, Plantago lanceolata, Blatella germanica, Acarus siro, Lepidoglyphus destructor, Tyrophagus putrescentiae e Dermatophagoides farinae. Utilizaram histamina $(10 \mathrm{mg} / \mathrm{ml})$ como controle positivo e solução salina como controle negativo. Os reagentes foram colocados

Tabela 1. Dados gerais e caracterização dos artigos selecionados, que utilizaram o teste cutâneo para sensibilização atópica em pacientes com asma e rinite alérgica.

\begin{tabular}{|c|c|c|c|c|c|c|c|c|}
\hline Autor & Ano & $\begin{array}{c}\text { Amostra } \\
\text { (n) }\end{array}$ & $\begin{array}{l}\text { Público } \\
\text { alvo }\end{array}$ & $\begin{array}{l}\text { Período } \\
\text { (coleta) }\end{array}$ & $\begin{array}{c}\text { País } \\
\text { (coleta) }\end{array}$ & $\begin{array}{l}\text { Alérgenos } \\
\text { (variedades) }\end{array}$ & $\begin{array}{c}\text { Local } \\
\text { (aplicado) }\end{array}$ & $\begin{array}{c}\text { Duração } \\
\text { (minutos) }\end{array}$ \\
\hline Bao-Qing [15] & 2010 & 6304 & $\mathrm{CR} / \mathrm{AD}$ & 2006-2007 & China & 16 & NC & NC \\
\hline Bousquet [16] & 2009 & 2928 & $\mathrm{AD}$ & NC & Europa & 18 & $\mathrm{NC}$ & 15 \\
\hline Comert [17] & 2014 & 7492 & $\mathrm{AD}$ & NC & Turquia & 20 & Antebraço & 15 \\
\hline Galván [18] & 2008 & 58 & $\mathrm{AD}$ & NC & México & 10 & Antebraço & 15 \\
\hline Karakaya [19] & 2012 & 169 & $\mathrm{AD}$ & 2011 & França & 9 & $\mathrm{NC}$ & 15 \\
\hline Makris [20] & 2009 & 316 & CR/AD & 2006-2008 & Grécia & 22 & NC & NC \\
\hline Migueres [21] & 2011 & 2714 & $\mathrm{CR}$ & 2008-2010 & França & 4 & Orelha & NC \\
\hline Misirlioğlu [22] & 2007 & 3025 & CR & NC & EUA & 36 & NC & NC \\
\hline Passalacqua [23] & 2013 & 318 & $C R / A D$ & NC & Itália & 14 & Antebraço & NC \\
\hline Pastorino [24] & 2008 & 1014 & $\mathrm{CR}$ & 2001-2002 & Brasil & 7 & Antebraço & NC \\
\hline Pérez [25] & 2009 & 258 & $\mathrm{AD}$ & 2006 & Cuba & 3 & Antebraço & 15 \\
\hline Rasool [26] & 2013 & 400 & $\mathrm{CR} / \mathrm{AD}$ & $\mathrm{NC}$ & Caxemira & 7 & NC & 20 \\
\hline Sahiner [27] & 2012 & 2457 & $\mathrm{CR}$ & 2008-2010 & Turquia & 30 & Tronco & 20 \\
\hline
\end{tabular}

AD: Adulto; CR: Criança; NC: Não consta. 
com espaço de $2 \mathrm{~cm}$ uns dos outros no antebraço do paciente, sendo utilizada uma lanceta com $1 \mathrm{~mm}$ de ponta para cada alérgeno, sendo pressionada no centro da gota do reagente sem provocar sangramento. Os resultados foram registrados após 15 minutos, considerando positivo os alérgenos e histamina com pápula $\geq 3 \mathrm{~mm}$ e a ausência de pápula no controle negativo. Os autores concluíram o estudo afirmando que não há necessidade de utilização de vários alérgenos para diagnóstico da sensibilização da asma ou rinite alérgica. Quatro dos vinte alérgenos testados possuem sensibilidade $>95 \%$ para tal finalidade [17].

Tabela 2. Identificação dos reagentes mais utilizados em testes cutâneos para o diagnóstico de asma e rinite alérgica.

Reagentes aplicados

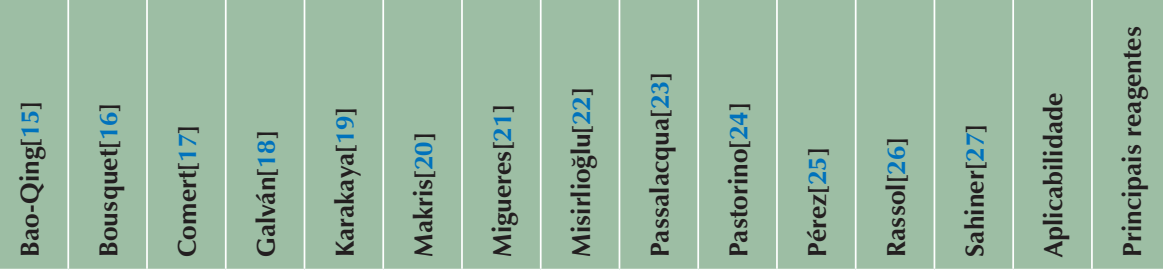

\begin{tabular}{|c|c|c|c|c|c|c|c|c|c|c|c|c|c|c|c|}
\hline Acarus siru & & & & $\mathrm{x}$ & & $\mathrm{x}$ & & & $\mathrm{x}$ & & & & & 3 & \\
\hline Alternaria & & $\mathrm{x}$ & $x$ & & & $x$ & & & $x$ & & & & $\mathrm{x}$ & 5 & \\
\hline Amaranthus spinosus & & & & & & & & & & & & $\mathrm{x}$ & & 1 & \\
\hline Ambrosia & & $\mathrm{x}$ & & & & & & & $\mathrm{x}$ & & & & & 2 & \\
\hline Artemisia vulgaris & & $\mathrm{x}$ & $\mathrm{x}$ & & $\mathrm{x}$ & $x$ & & & $\mathrm{x}$ & & & & $\mathrm{x}$ & 6 & * \\
\hline Aspergillus fumigatus & & $x$ & $\mathrm{x}$ & & & $\mathrm{x}$ & & & $x$ & & & & & 4 & \\
\hline Bétula & & $\mathrm{x}$ & & & $\mathrm{x}$ & $x$ & & & $\mathrm{x}$ & & & & & 4 & \\
\hline Blattella germanica & $x$ & $\mathrm{x}$ & $\mathrm{x}$ & $\mathrm{x}$ & & & & & & $x$ & & & & 5 & \\
\hline Blomia tropicalis & & & & $x$ & & & & & & & & & & 1 & \\
\hline Chenopodium mixture & & & & & & & & & & & & & $\mathrm{x}$ & 1 & \\
\hline Cipreste & & $x$ & & & & & & & $x$ & & & & & 2 & \\
\hline Cladosporium herbarum & & $\mathrm{x}$ & $\mathrm{x}$ & & & $x$ & & & & & & & $\mathrm{x}$ & 4 & \\
\hline Coockroach & & & & & & & & $\mathrm{x}$ & & & & & $\mathrm{x}$ & 2 & \\
\hline Corylus avellana & & & & & $x$ & & & & & & & & & 1 & \\
\hline Cynodon dactylon & & & & & & $x$ & & & & & $\mathrm{x}$ & $x$ & $\mathrm{x}$ & 4 & \\
\hline Dermatophagoides farinae & & $x$ & $\mathrm{x}$ & $\mathrm{x}$ & & $x$ & & $x$ & & & & & $x$ & 6 & * \\
\hline Dermatophagoides glomerata & & & & & & & & & & & & & $\mathrm{x}$ & 1 & \\
\hline Dermatophagoides pteronyssinus & & $\mathrm{x}$ & $\mathrm{x}$ & $\mathrm{x}$ & $\mathrm{x}$ & $\mathrm{x}$ & & $\mathrm{x}$ & & $\mathrm{x}$ & & $\mathrm{x}$ & $\mathrm{x}$ & 9 & * \\
\hline Epitélio de cão & & $\mathrm{x}$ & $\mathrm{x}$ & & $x$ & $\mathrm{x}$ & & $x$ & $\mathrm{x}$ & $\mathrm{x}$ & & & $\mathrm{x}$ & 8 & * \\
\hline Epitélio de gato & & $\mathrm{x}$ & $x$ & & $\mathrm{x}$ & $x$ & $\mathrm{x}$ & $\mathrm{x}$ & $\mathrm{x}$ & $\mathrm{x}$ & & & $\mathrm{x}$ & 9 & * \\
\hline Grama Timothy & & $x$ & & & & & & & & & & & & 1 & \\
\hline Gramíneas & & $x$ & & & & & $x$ & & $\mathrm{x}$ & $\mathrm{x}$ & & & & 4 & \\
\hline Lepidoglyphus destructor & & & & $\mathrm{x}$ & & & & & & & & & & 1 & \\
\hline Lolium por & & & & & & $x$ & & & & & & & & 1 & \\
\hline Olea europea & & & $\mathrm{x}$ & & $\mathrm{x}$ & $x$ & & & & & & & & 3 & \\
\hline Oliveira & & $\mathrm{x}$ & $\mathrm{x}$ & & & & & & $x$ & & & & & 3 & \\
\hline Parietaria & & $\mathrm{x}$ & $\mathrm{x}$ & & $\mathrm{x}$ & $\mathrm{x}$ & & & $\mathrm{x}$ & & & & & 5 & \\
\hline Parténio hysterophorus & & & & & & & & & & & & $x$ & & 1 & \\
\hline Penicillium & & & & & & $\mathrm{x}$ & & & & & & & $\mathrm{x}$ & 2 & \\
\hline Periplaneta americana & $\mathrm{x}$ & & $\mathrm{x}$ & $\mathrm{x}$ & & & & & & $\mathrm{x}$ & & & & 4 & \\
\hline Periplaneta fuliginosa & $x$ & & & & & & & & & & & & & 1 & \\
\hline Plátano & & $\mathrm{x}$ & & & & $x$ & & & & & & & & 2 & \\
\hline Prado de aveia & & $x$ & & & & & & & & & & & & 1 & \\
\hline Pratense phleum & & & $\mathrm{x}$ & & $\mathrm{x}$ & & & & & & & & $\mathrm{x}$ & 3 & \\
\hline Secale cer & & & & & & $\mathrm{x}$ & & & & & & & & 1 & \\
\hline Taraxacum vulg & & & & & & $\mathrm{x}$ & & & & & & & & 1 & \\
\hline Total de reagentes positivos & 3 & 18 & 14 & 7 & 9 & 18 & 2 & 5 & 12 & 6 & 1 & 4 & 13 & & \\
\hline
\end{tabular}

* Cinco principais reagentes aplicados nos estudos. 
Galván et al. [18] desenvolveram um estudo em três cidades do México, com objetivo de avaliar a sensibilização dos pacientes a alérgenos e determinar se apresentavam diferença em relação a condições climáticas e geográficas. Aplicaram o teste cutâneo em 58 pacientes, testando oito tipos de ácaros e duas espécies de baratas, sendo eles: Dermatophagoides pteronysinnus, Dermatophagoides farinae, Blomia tropicalis, Tyrophagus putrescientiae, Lepidoglyphus destructor, Euroglyphus maynei, Acarus siro, Dermatophagoides microceras, Periplaneta americana e Blattella germânica, tendo como resultado maior sensibilização a baratas, independente das condiçoes geográficas e climáticas [18].

Karakaya et al. [19] desenvolveram um estudo com o objetivo de avaliar a taxa de predição de sucesso de atopia em 169 pacientes com asma e rinite alérgica. Aplicaram o teste cutâneo com os seguintes reagentes: Dermatophagoides pteronyssinus, Phleum pratense, Olea europea, Artemisia vulgaris, Parietaria officinalis, Corylus avellana, Betula verrucosa, epitélios de gato e cão. A previsão de positividade para os testes era de $42,6 \%$, porém resultou em $36,1 \%$. Com o ponto de corte em $\geq 3 \mathrm{~mm}$, a previsão de sensibilização foi de $77 \%$, com especificidade de $65,3 \%$. Com o limite de $5 \mathrm{~mm}$ para um teste positivo, a sensibilização, a especificidade, os valores positivos e negativos foram de $91 \%, 61 \%, 14 \%$ e $99 \%$, respectivamente [19].

Makris at al. [20] desenvolveram um estudo com objetivo de estimar um padrão clínico de rinite e estipular sua relação com a asma em uma população grega. Avaliaram 316 pacientes, sendo aplicados os seguintes alérgenos: Dermatophagoides pteronyssinus, Dermatophagoides farinae, Phleum pr, Cynodon D, Lolium per, Secale cer, Parietaria off, Artemisia vul, Taraxacum Vulg, Plantago lan, Olea Europea, Platanus, Betula, Quercus, Fagus, Poplar, epitélio de gato, epitélio de cão, Cladosporium, Penicillium, Alternaria Alt, Aspergillus mix. Utilizaram histamina $(10 \mathrm{mg} / \mathrm{ml})$ como controle positivo e solução salina como controle negativo. Os autores não especificaram o local de aplicação, tempo de duração do teste e tipo de lanceta utilizada. Consideraram positivo os alérgenos e histamina com pápula $\geq 3 \mathrm{~mm}$ e a ausência de pápula no controle negativo. Concluíram o estudo afirmando que mais de $60 \%$ dos pacientes estudados possuem sensibilização a um ou mais alérgenos sazonais, com Parietaria off sendo o alérgeno mais comum [20].

Migueres et al. [21] desenvolveram um estudo na França com crianças com asma e/ou rinite alérgica, com o objetivo de avaliar a sensibilização de quatro variedades de alérgenos. Participaram do estudo 2.714 pacientes, sendo constatado que $64,5 \%$ dos pacientes obtiveram reação a ácaros, $61,5 \%$ ao pólen de flores, $41,6 \%$ ao pólen de árvore e $30,5 \%$ ao epitélio de gato [21].

Misirlioğlu et al. [22] desenvolveram um estudo com crianças de três meses a 16 anos, em localidades dos EUA, com o objetivo de avaliar a sensibilização a alérgenos usando teste cutâneo em pacientes com diagnóstico de asma brônquica. Foram testados 36 tipos de alérgenos diferentes em 3.025 pacientes, sendo que $60,3 \%$ desses pacientes foram considerados atópicos. Em 26,9\% dos pacientes foram diagnosticados alergia de pele de animais e caspa, sendo os alérgenos testados: cachorro, gato, pássaros e ovinos. Para baratas, a porcentagem de pacientes com alergia foi de $16,3 \%$. Foram também testadas 18 variedades de alérgenos de comida, dando ênfase a clara de ovos e cacau, em um total de $11,3 \%$ de pacientes atópicos. Em $63,3 \%$ dos pacientes testados foi identificada alergia a ácaros, sendo os alérgenos testados: Dermatophagoide farinae e Dermatophagoide pteronyssinus. Os pólens foram detectados em 49,3\% dos pacientes. Alergia a gramíneas aparece em 30,3\%, sendo a Graminnea o alérgeno testado. Foram testadas seis variedades de alérgenos a pólen de árvore, com $16,5 \%$ de positividade. Para plantas de ervas daninhas foram testados dois tipos de alérgenos, com $23,9 \%$ de positividade. Por fim, alergia ao látex foi detectada em 3,6\% dos casos. Não identificaram o local onde foi aplicado o teste cutâneo, somente informaram a aplicação na pele e a reação foi avaliada 20 minutos após, sendo utilizada uma lanceta metálica tipo A [22].

Passalacqua et al. [23] avaliaram as informações de diagnóstico adicional fornecidas por um microarray alérgeno em uma grande população de indivíduos polissensibilizados. Aplicaram o teste cutâneo em 318 pacientes, cujos alérgenos foram: ácaros, grama, oliveira, Parietaria, bétula, cipreste, ambrósia, artemísia, epitélio de gato e cachorro, Alternaria e Aspergillus. Utilizaram histamina como controle positivo e o diluente como controle negativo. Consideraram como teste positivo pápula com reação $\geq 3 \mathrm{~mm}$. Nenhuma preparação de alérgeno recombinante foi utilizada neste diagnóstico. Concluíram o estudo afirmando que Aspergillus, Parietaria e componentes de grama, foram identificados em torno de $20 \%$ dos indivíduos [23].

Pastorino et al. [24] desenvolveram um estudo com 1.014 adolescentes brasileiros matriculados em escolas públicas na região oeste da cidade de São Paulo/SP e na cidade de Nova Iguaçu/RJ. O objetivo foi avaliar e comparar a sensibilidade desses 
adolescentes a aeroalérgenos, com a aplicação do teste cutâneo, correlacionando seu resultado positivo com o diagnóstico de asma e rinite. Os reagentes utilizados foram: controle positivo (10 $\mathrm{mg} / \mathrm{ml}$ de histamina) e controle negativo (solução de fenol a $0,5 \%$ e solução de glicerina). Os alérgenos foram Dermatophagoides pteronyssinus, Periplaneta americana, Blattella germanica, epitélio de cão e gato, e uma mistura de fungos e grama. Concluíram o estudo obtendo como resultado uma alta taxa de sensibilização a aeroalérgenos, significativamente maior entre aqueles com asma ou rinite, ou uma combinação de ambos, especialmente em múltiplas sensibilizações [24].

Pérez et al. [25] desenvolveram um estudo com objetivo de avaliar a eficácia do teste cutâneo com extrato de Cynodon dactylon em asmáticos e portadores de rinite. Aplicaram o teste cutâneo em 204 pacientes e 54 indivíduos saudáveis, sendo os seguintes alérgenos: extrato de pólen alergênico Cynodon dactylon, histamina como controle positivo e solução salina como controle negativo. $O$ teste foi realizado no antebraço, e os reagentes foram colocados a $2 \mathrm{~cm}$ de distância uns dos outros. Uma gota de cada substância foi aplicada nos pontos marcados por inserção de $1 \mathrm{~mm}$ de lanceta, com uma inclinação de $45^{\circ}$ em relação à pele. Os resultados foram obtidos 15 minutos após a aplicação do teste, concluindo que a rinite alérgica foi à doença mais prevalente, sendo que $62,9 \%$ dos pacientes eram sensibilizados ao extrato de Cynodon dactylon [25].

Rasool et al. [26] desenvolveram um estudo com 400 pacientes com rinite alérgica, asma e urticária, sendo a maioria moradores da Caxemira. A faixa etária variou dos seis anos até os 65 anos. Dentre os 400 pacientes, 248 tinham urticária, 108 eram portadores de rinite alérgica e 44 pacientes tinham asma. A reação cutânea foi lida após 20 minutos e considerada positiva quando o diâmetro da pápula fosse pelo menos 3 $\mathrm{mm}$ maior do que o controle negativo. Encontraram respostas positivas mais comuns ao pólen $(52 \%)$, seguido do ácaro doméstico (44\%), poeiras $(2 \%)$, baratas $(1,5 \%)$ e fungos $(0,5 \%)$. Concluíram que na Caxemira o alérgeno mais comum foi o pólen, seguido por ácaros da espécie Dermatophagoides pteronyssinus, enquanto os ovinos, fungos, cães, e gatos são incomuns nesta região. A imunoterapia foi eficaz na redução dos sintomas graves em 58\% dos pacientes com rinite alérgica e $42 \%$ dos pacientes com asma [26].

Sahiner at al.[27] desenvolveram um estudo com o objetivo de avaliar os padrões de sensibilização entre crianças de diferentes faixas etárias, e definir tanto o número quanto o tipo de alérgenos necessários para identificar uma criança sensibilizada. Aplicaram o teste cutâneo em 2.457 pacientes, com os seguintes alérgenos: Dermatophagoides pteronyssinus, Dermatophagoides farinae, Blattella germanica, epitélio de gato, cavalo e cão, Alternaria alternata, Aspergillus fumigatus, Penicilium mistura (P. digitatum, $P$. expansume, $P$. notatum), Cladosporium herbarum, Phleum pratense, Poa pratensis, Dactylis glomerata, Lolium perenne, Festuca pratensis, Avena sativa, Cynodon dactylon, Parietaria judaica, Artemisia vulgaris, Plantago, Chenopodium mistura, Salsola kali, Salix caprea, Ulmus campestris, Quercus robur, avelã, Betula alba, Populus alba, Platanus vulgaris e Olea europaea. Utilizaram histamina $(10 \mathrm{mg} / \mathrm{ml})$ como controle positivo e $0,9 \%$ de solução salina estéril como controle negativo. Os autores não especificaram o local de aplicação, tempo de duração do teste e tipo de lanceta utilizada. Os resultados foram registrados após 20 minutos, considerando positivo se o diâmetro da pápula foi $\geq 3 \mathrm{~mm}$ em comparação com o controle negativo. Os autores concluíram que o alérgeno mais comum foi gramínea, seguido por ácaros, gato, erva daninha e Alternaria. Testando com F. pratensis, Dermatophagoides pteronyssinus, P. pratense, Alternaria e gato identificou-se cerca de $90 \%$ de sensibilização. Para chegar a uma taxa de sensibilização de $95 \%$, nove alérgenos se mostraram suficientes [27].

\section{DISCUSSÃO}

O teste cutâneo é um método amplamente utilizado na área clínica para análise da sensibilização a determinados antígenos, auxiliando no diagnóstico de asma ou rinite alérgica [28]. O surgimento de pápula representa uma reação em que há presença do anticorpo IgE específico em relação ao alérgeno testado [29]. No entanto, para ser considerado positivo, deve-se aplicar em conjunto o controle negativo (solução salina) e o controle positivo (histamina), considerando positivo o resultado cujo diâmetro da pápula for igual ou maior a três milímetros em relação ao controle positivo [8]. O significado do teste positivo relaciona-se a uma sensibilização e não necessariamente à alergia, sendo necessária uma correlação clínica para comprovação da doença alérgica [29].

O teste cutâneo apresenta valores positivos entre 10 e $15 \%$ de pacientes assintomáticos, que porventura podem vir apresentar sintomas da doença alérgica com o passar dos anos. Cerca de $50 \%$ das crianças assintomáticas que apresentam positividade no teste 
cutâneo, apresentam risco de desenvolver rinite durante os próximos cinco anos [28].

A principal vantagem do este cutâneo é a praticidade e rapidez do resultado, que é dado entre 30 a 40 minutos [29], além de possuir baixa incidência de efeitos adversos e baixo custo [31]. Em estudos clínicos, além de servir como fator de identificação a sensibilizações, serve como avaliação da resposta a tratamentos para atopia, como, por exemplo, a imunoterapia [30] e em programas de educação em doenças alérgicas, fazendo com que o paciente visualize a resposta alérgica, reforçando a informação verbal e ajudando na avaliação da causa-efeito da alergia. Medidas de controle ambiental devem ser mantidas em conjunto com a prevenção da sensibilização alérgica e na redução de manifestações clínicas em pacientes com asma ou rinite alérgica [32]. O desafio, segundo as diretrizes, é garantir a adesão dos indivíduos no manejo do tratamento para o controle efetivo da doença [33,34]. Contudo, no que se refere ao teste cutâneo, o principal desafio para o diagnóstico preciso vai além da aplicação precisa, em virtude da sensibilização variar de indivíduo para indivíduo [35]. Inúmeros são os reagentes específicos testados mundialmente, não havendo um consenso sobre quais são os principais alérgenos que devam ser aplicados nos diagnósticos de asma e rinite alérgica [17], dificultando a escolha dos reagentes mais adequados para a aplicação.

Dos 36 alérgenos aplicados nos estudos incluídos nesta revisão, cinco deles possuíram maior frequência de aplicabilidade e sensibilização (Artemisia vulgaris, Dermatophagoides farinae, Dermatophagoides pteronyssinus e epitélios de cão e gato). Os estudos mostram variedade na frequência de positividade a alérgenos, de acordo com a região e população em que o teste é aplicado.
Três dos 13 estudos [16, 17, 23] analisaram a sensibilidade e especificidade dos alérgenos, na tentativa de identificar os mais aplicáveis nos testes cutâneos. Bousquet et al. [16] analisaram 18 alérgenos e concluíram que oito são suficientes para identificação de mais de $95 \%$ dos indivíduos sensibilizados. Já Comert et al. [17] aplicaram 20 alérgenos e concluíram afirmando que quatro reagentes são suficientes para identificação de $95 \%$ dos sensibilizados. Contudo, Sahiner at al. [23] também aplicaram 20 tipos de alérgenos, chegando à conclusão que para uma precisão superior a 95\%, nove tipos são necessários para esta taxa de detecção. Dos 13 artigos analisados, os valores de positividade à sensibilização variaram de $0,5 \%$ para fungos a $68,2 \%$ para gramíneas, sendo que os mais comuns foram para os extratos de ácaros (Dermatophagoides farinae e Dermatophagoides pteronyssinus), com valores médios acima dos $60 \%$.

Como limitação desta revisão sistemática, apontamos a falta de acesso a 10 artigos na íntegra, não estando disponíveis via portal CAPES, nem pela biblioteca central da Instituição afiliada.

\section{CONSIDERAÇÕES FINAIS}

Para que haja uma boa confiabilidade na aplicação de testes de sensibilização para atopia, o método de aplicação deve ser padronizado, devendo ser estudados os tipos mais característicos da região investigada. Além disso, o teste cutâneo não deve ser utilizado isoladamente para fins de diagnóstico da doença alérgica, sendo um exame adjuvante na determinação do componente atópico e auxiliar no manejo do controle ambiental.

\section{REFERÊNCIAS}

1. Hosseini S, Shoormasti RS, Akramian R, Movahedi M, Gharagozlou M, Foroughi N, Saboury B, Kazemnejad A, Mahlooji Rad M, Mahdaviani A, Pourpak Z, Moin M. Skin Prick Test Reactivity to Common Aero and Food Allergens among Children with Allergy. Iran J Med Sci. 2014;39(1):29-35.

2. Ege MJ, Mayer M, Normand AC, Genuneit J, Cookson WO, Braun-Fahrländer C, Heederik D, Piarroux R, von Mutius E; GABRIELA Transregio 22 Study Group. Exposure to environmental microorganisms and childhood asthma. N Engl J Med. 2011;364(8):701-9. http:// dx.doi.org/10.1056/NEJMoa1007302

3. Newson RB, Jones M, Forsberg B, Janson C, Bossios A, Dahlen SE, Toskala EM, Al-Kalemji A, Kowalski ML, Rymarczyk B, Salagean EM, van Drunen CM, Bachert C, Wehrend T, Krämer U, Mota-Pinto A, Burney P, Leynaert B, Jarvis D. The association of asthma, nasal allergies, and positive skin prick tests with obesity, leptin, and adiponectin. Clin Exp Allergy. 2014;44(2):250-60. http://dx.doi.org/10.1111/ cea. 12221

4. Awasthi A, Singh R. Determination of aerobiological flora associated with allergic rhinitis by Skin Prick Test in a tertiary care hospital in the West Coast of Southern India. Clin Epid Glob Heal. 2014;2(3):143-8. http://dx.doi.org/10.1016/j.cegh.2014.08.003

5. Payot F, Berthiller J, Kassai B, Brunet A-S, Villard-Truc F, Lachaux A. Practical interest of both skin prick test and specific IgE in the evaluation of tolerance acquisition in IgE mediated cow's milk allergy (CMA). A clinical retrospective study in a cohort of 184 children. Allergol Immunopathol (Madr). 2014;42(5):395-401. http://dx.doi.org/10.1016/j.aller.2013.03.007 
6. Haahtela T1, Burbach GJ, Bachert C, Bindslev-Jensen C, Bonini S, Bousquet J, Bousquet-Rouanet L, Bousquet PJ, Bresciani M, Bruno A, Canonica GW, Darsow U, Demoly P, Durham SR, Fokkens WJ, Giavi S, Gjomarkaj M, Gramiccioni C, Kowalski ML, Losonczy G, Orosz M, Papadopoulos NG, Stingl G, Todo-Bom A, von Mutius E, Köhli A, Wöhrl S, Järvenpää S, Kautiainen H, Petman L, Selroos O, Zuberbier T, Heinzerling LM. Clinical relevance is associated with allergen-specific wheal size in skin prick testing. Clin Exp Allergy. 2014;44(3):407-16. http://dx.doi.org/10.1111/cea.12240

7. Norrman G1, Fälth-Magnusson K. Adverse reactions to skin prick testing in children-prevalence and possible risk factors. Pediatr Allergy Immunol. 2009;20(3):273-8. http://dx.doi.org/10.1111/j.1399-3038.2008.00761.x

8. Bousquet J, Heinzerling L, Bachert C, Papadopoulos NG, Bousquet PJ, Burney PG, Canonica GW, Carlsen KH, Cox L, Haahtela T, Lodrup Carlsen KC, Price D, Samolinski B, Simons FE, Wickman M, Annesi-Maesano I, Baena-Cagnani CE, Bergmann KC, Bindslev-Jensen C, Casale TB, Chiriac A, Cruz AA, Dubakiene R, Durham SR, Fokkens WJ, Gerth-van-Wijk R, Kalayci O, Kowalski ML, Mari A, Mullol J, Nazamova-Baranova L, O’Hehir RE, Ohta K, Panzner P, Passalacqua G, Ring J, Rogala B, Romano A, Ryan D, Schmid-Grendelmeier P, Todo-Bom A, Valenta R, Woehrl S, Yusuf OM, Zuberbier T, Demoly P; Global Allergy and Asthma European Network; Allergic Rhinitis and its Impact on Asthma. Practical guide to skin prick tests in allergy to aeroallergens. Allergy. 2012;67(1):18-24. http://dx.doi. org/10.1111/j.1398-9995.2011.02728.x

9. Sekerel BE, Sahiner UM, Bousquet J, Demoly P, Zuberbier T, Carlsen KH, Wickman M. Practical guide to skin prick tests in allergy to aeroallergens: some concerns. Allergy. 2012;67(3):442; author reply 443. http://dx.doi.org/10.1111/j.1398-9995.2012.02772.x

10. Lee S, Kundaria S, Ferguson BJ. Practical clinical management strategies for the allergic patient with chronic rhinosinusitis. Curr Opin Otolaryngol Head Neck Surg. 2012;20(3):179-87. http://dx.doi.org/10.1097/MOO.0b013e328352b839

11. Heinzerling L, Mari A, Bergmann KC, Bresciani M, Burbach G, Darsow U, Durham S, Fokkens W, Gjomarkaj M, Haahtela T, Bom AT, Wöhrl S, Maibach H, Lockey R.The skin prick test-European standards. Clin Transl Allergy. 2013;3(1):3. http://dx.doi.org/10.1186/20457022-3-3

12. Vázquez-Ortiz M, Alvaro-Lozano M, Alsina L, Garcia-Paba MB, Piquer-Gibert M, Giner-Mu-oz MT, Lozano J, Domínguez-Sánchez O, Jiménez R, Días M, Martín-Mateos MA, Plaza-Martín AM. Safety and predictors of adverse events during oral immunotherapy for milk allergy: severity of reaction at oral challenge, specific IgE and prick test. Clin Exp Allergy. 2013;43(1):92-102. http://dx.doi.org/10.1111/ cea. 12012

13. Zamboni A, Di Thommazo A, Hernandes E, Fabbri S, editors. StArt Uma Ferramenta Computacional de Apoio à Revisão Sistemática. In: Congresso Brasileiro de Software (CBSoft'10). Salvador, Brazil; 2010.

14. Guyatt GH, Oxman AD, Schünemann HJ, Tugwell P, Knottnerus A. GRADE guidelines: A new series of articles. J Clin Epidemiol. 2011;64(4):380-2. http://dx.doi.org/10.1016/j.jclinepi.2010.09.011

15. Sun B-q, Lai X-x, Gjesing B, Dho Spangfort M, Zhong N-s. Prevalence of sensitivity to cockroach allergens and IgE cross-reactivity between cockroach and house dust mite allergens in Chinese patients with allergic rhinitis and asthma. Chin Med J (Engl). 2010;123(24): $3540-4$.

16. Bousquet PJ, Burbach G, Heinzerling LM, Edenharter G, Bachert C, Bindslev-Jensen C, Bonini S, Bousquet-Rouanet L, Demoly P, Bresciani M, Bruno A, Gjomarkaj M, Canonica GW, Darsow U, Durham S, Fokkens WJ, Giavi S, Gramiccioni C, Papadopoulos NG, Haahtela T, Kowalski ML, Magyar P, Muraközi G, Orosz M, Röhnelt C, Stingl G, Todo-Bom A, von Mutius E, Wiesner A, Wöhrl S, Bousquet J, Zuberbier T. GA2LEN skin test study III: minimum battery of test inhalent allergens needed in epidemiological studies in patients. Allergy. 2009;64(11):1656-62. http://dx.doi.org/10.1111/j.1398-9995.2009.02169.x

17. Comert S, Demir AU, Karakaya G, Kalyoncu AF. Minimum prick test panel for adult patients with asthma and rhinitis in Ankara, Turkey. J Asthma. 2014;51(4):417-22. http://dx.doi.org/10.3109/02770903.2013.878846

18. Cavazos Galván M, Guerrero Nú-ez B, Ramírez Aragón D. Comparative mites and cockroaches sensitization study in three cities of Mexico. Rev Alerg Mex. 2008;55(6):234-9.

19. Karakaya G, Ozturk AB, Kalyoncu AF. Prediction of atopy by skin prick tests in patients with asthma and/or persistent rhinitis. Allergol Immunopathol (Madr). 2012;40(1):37-40. http://dx.doi.org/10.1016/j.aller.2011.01.005

20. Makris M, Koulouris S, Koti I, Aggelides X, Sideri K, Chliva C, Vassilatou E, Kalogeromitros D. Temporal relationship of allergic rhinitis with asthma and other co-morbidities in a Mediterranean country: a retrospective study in a tertiary reference allergy clinic. Allergol Immunopathol (Madr). 2010;38(5):246-53. http://dx.doi.org/10.1016/j.aller.2009.11.007

21. Migueres M, Fontaine JF, Haddad T, Grosclaude M, Saint-Martin F, Bem David D, Crestani B. Characteristics of patients with respiratory allergy in France and factors influencing immunotherapy prescription: a prospective observational study (REALIS). Inter J Immun Pharm. 2010;24(2):387-400

22. Dibek Misirlioğlu E, Reha Cengizlier M. Skin prick test results of child patients diagnosed as bronchial asthma. Allergol Immunopathol (Madr). 2007;35(1):21-4. http://dx.doi.org/10.1157/13099091

23. Passalacqua G, Melioli G, Bonifazi F, Bonini S, Maggi E, Senna G, Triggiani M, Nettis E, Rossi RE, Vacca A, Canonica GW; Italian ISAC Study Group. The additional values of microarray allergen assay in the management of polysensitized patients with respiratory allergy. Allergy. 2013;68(8):1029-33. http://dx.doi.org/10.1111/all.12194

24. Pastorino AC, Kuschnir FC, Arruda LK, Casagrande RR, de Souza RG, Dias GA, Silveira HH, da Cunha AJ, Jacob CM, Solé D. Sensitisation to aeroallergens in Brazilian adolescents living at the periphery of large subtropical urban centres. Allergol Immunopathol (Madr). 2008;36(1):9-16. http://dx.doi.org/10.1157/13115665

25. Pérez JR, Ramos CMS, Fabelo MP, Candelario ZG. Ensayo clínico diagnóstico con extracto alergénico de polen de Cynodon dactylon en pacientes con asma y/o rinitis. Rev Arch Med Camagüey. 2009;13(4):1-8.

26. Rasool R, Shera IA, Nissar S, Shah ZA, Nayak N, Siddiqi MA, Sameer AS.Role of skin prick test in allergic disorders: A prospective study in Kashmiri population in light of review. Indian J Dermatol. 2013;58(1):12-7. http://dx.doi.org/10.4103/0019-5154.105276 
27. Şahiner UM1, Civelek E, Yavuz ST, Büyüktiryaki AB, Tuncer A, Şekerel BE. Skin prick testing to aeroallergen extracts: what is the optimal panel in children and adolescents in Turkey? Int Arch Allergy Immunol. 2012;157(4):391-8. http://dx.doi.org/10.1159/000329870

28. Sicherer SH, Wood RA; American Academy of Pediatrics Section On Allergy And Immunology. Allergy testing in childhood: using allergen-specific IgE tests. Pediatrics. 2012;129(1):193-7. http://dx.doi.org/10.1542/peds.2011-2382

29. Greiner AN, Hellings PW, Rotiroti G, Scadding GK. Allergic rhinitis. The Lancet. 2011;378(9809):2112-22. http://dx.doi.org/10.1016/ S0140-6736(11)60130-X

30. Yukselen A, Kendirli SG, Yilmaz M, Altintas DU, Karakoc GB. Effect of one-year subcutaneous and sublingual immunotherapy on clinical and laboratory parameters in children with rhinitis and asthma: a randomized, placebo-controlled, double-blind, double-dummy study. Int Arch Allergy Immunol. 2012;157(3):288-98. http://dx.doi.org/10.1159/000327566

31. Columbo M, Wong B, Panettieri Jr RA, Rohr AS. The effect of multiple allergen immunotherapy on exhaled nitric oxide in adults with allergic rhinitis. Allergy Asthma Clin Immunol. 2013;9(1):31 http://dx.doi.org/10.1186/1710-1492-9-31

32. Trinca MA, Bicudo IM, Pelicioni MCF. A interferência da asma no cotidiano das crianças. Rev Bras Cresc Desenv Hum. 2011;21(1): $70-84$.

33. Diretrizes da Sociedade Brasileira de Pneumologia e Tisiologia para o manejo da asma-2012. J Bras Pneumol. 2012;38 Suppl 1:S1-S46.

34. de Castro GR, Lima C, da Silva EV, de Toledo Nóbrega O, Naves JOS. Seguimento das diretrizes terapêuticas e adesão à farmacoterapia no tratamento da asma. Brasília Med. 2013;50(3):206-12.

35. Dias JG, Silva SL, Luz S, Silva SP, Santos AS, Pedro E. Estudo comparativo de métodos de rastreio de atopia em doentes com rinite (ImmunoCAP ${ }^{\circledR}$ Rapid versus Phadiatop ${ }^{\circledR}$ e testes cutâneos em picada). Rev Portug Imunoalerg. 2013;21(1):41-7. 